\title{
THE DIAGNOSIS OF EPILEPSY BEGINNING IN ADULT LIFE.
}

\author{
By W. RUSSELL BRAIN, D.M., F.R.C.P.
}

(Physician with Charge of Out-patients to the London Hospital, Physician to the Maida Vale $\stackrel{\overrightarrow{\overrightarrow{\vec{S}}}}{\stackrel{c}{+}}$ Hospital for Nervous Diseases.)

The large majority of patients suffering from epileptic attacks begin to have $\frac{\Phi}{\triangle}$ fits before the age of twenty. Nevertheless the minority in whom convulsions $\%$ first appear after this age is an important one, because most persons who are $\vec{P}$ younger than twenty when the attacks begin are suffering from idiopathic epilepsy, the treatment of which is symptomatic. Idiopathic epilepsy, however, rarely $\vec{\omega}$ begins after the age of twenty-five. It follows, therefore, that most persons who $\frac{\text { s }}{8}$ begin to have fits in adult life are suffering from symptomatic epilepsy. Such patients require a very careful and complete investigation if the cause of their attacks is to be discovered. The investigation must begin with a detailed history $\vec{P}$ of the attacks, including not only the patient's account but that of an eye-witness, $\vec{v}$ since the details of the attack are necessary both for the diagnosis of its nature, and also for the localization of a lesion of the brain when this is the cause. It $\vec{\sigma}$ is of special importance to ascertain the exact nature of the aura which ushers 9 in the attack if one occurs, the distribution of any involuntary movements which $\rightarrow$ are seen in the attack, especially at its onset, and the character and duration of any $\frac{7}{0}$ residual symptoms, such as difficulty in speaking, or weakness of a limb.

Since an epileptic attack consists essentially in an abnormal discharge neural energy which may originate in any part of the cerebral hemispheres, large variety of epileptic attacks are recognized, corresponding to the site of origin and extent of spread of the abnormal discharge. Hence, in addition to the familiar major and minor attacks we encounter other varieties of seizure. In Jacksonian epilepsy the attacks begin with clonic movements limited to a small $\stackrel{\circ}{\curvearrowright}$ segment of the body owing to the excitation of the corresponding area of the $\overrightarrow{\vec{D}}$ opposite pre-central cortex. As the neural discharge spreads through adjacent 3 cortical areas the convulsions similarly spread to corresponding parts of the body. There is also sensory Jacksonian epilepsy in which the attacks begin with a sensory disturbance which spreads in the same way. Uncinate attacks, which are caused o by excitation of the uncinate gyrus, begin with an hallucination of taste or smell, which is followed by impairment of consciousness and convulsive movements of the lips, tongue and jaw.

An account of the attacks having been obtained, enquiry is made as to other symptoms, especially headache, vomiting, visual impairment, mental deterioration and speech disorder. A careful examination of the ocular fundi, the central nervous and cardio-vascular systems, the blood pressure and the urine concludes the clinical investigation. In most cases further examinations will be necessary. 0 Lumbar puncture will enable the pressure of the cerebro-spinal fluid to be recorded $\widetilde{N}$ and the fluid itself to be examined. The Wassermann reaction should be done in $\underset{\omega}{N}$ both the blood and the cerebro-spinal fluid and if it is positive in the latter the colloidal gold test should also be carried out. Routine stereoscopic radiograms of $o$ the skull are necessary in all cases in which an intracranial tumour is suspected, since these may demonstrate, amongst other things, erosion of the bones of the skull or calcification within a tumour. It will sometimes be necessary to 0 take further radiograms after filling the ventricles with air injected into the sub- $\frac{0}{\pi}$ arachnoid space by lumbar puncture. This process is known as encephalography 
and enables abnormalities in the size, shape and position of the ventricles to be visualized.

It has recently been found possible to amplify and record the minute changes in electrical potential occurring in the cerebral cortex by means of an apparatus applied to the scalp. This process, known as electro-encephalography, has already demonstrated that abnormal cortical electrical rhythms form the basis of an epileptic attack and promises to be of increasing value in diagnosis.

\section{Intracranial Tumour.}

Intracranial tumour is a sufficiently common cause of convulsions to be considered in every case of epilepsy beginning in adult life. Sachs in a series of 724 cases of intracranial tumour found that I patient in every 5 had convulsions. The proportion was even higher, $I$ in 3 , of patients in whom the tumour involved one or other cerebral hemisphere. Since tumours of the cerebral hemispheres are very rare in childhood almost all the patients with intracranial tumours who suffer from epilepsy are adults. Whereas in many such cases the familiar symptoms and signs of increased intracranial pressure, such as headache, vomiting and papillœdema, together with focal signs of the tumour, will be present, it is very important to bear in mind that this is not always the case but that epilepsy may precede all other symptoms and signs of an intracranial tumour by many years. Sachs found that I8 per cent. of his patients who had convulsions gave a history of fits over a period ranging from 5 to 25 years before the diagnosis of tumour was made. One of my own patients suffered from epilepsy for I7 years before developing any other symptoms or signs of a large glioma of his left cerebral hemisphere. The convulsions are usually generalized, irrespective of the situation of the tumour. Jacksonian convulsions are rare and occur only when the tumour involves the pre-central convolution. Tumours in the neighbourhood of the tip of the temporal lobe may cause the characteristic uncinate attacks which have already been described. The following two cases illustrate these points:-

A bank manager, aged 52, in 1932 had an epileptic fit. This was followed by occasional minor epileptic attacks and some impairment of memory. In 1934 he had another major convulsion and after this his memory became worse. When first seen in 1935 he made no complaint of headache. His ocular fundi were normal and there was no abnormality in the nervous system. An encephalogram was carried out and it was found that the left cerebral ventricle did not fill as well with air as the right. This was suggestive of a tumour in the substance of the left hemisphere, but in view of the absence of symptoms of increased intracranial pressure and the probability that an operation in this region would seriously damage the speech function, it was decided to await events. Later, in 1935, the character of the epileptic attacks changed somewhat as they were then preceded by hallucinations of taste or smell. In March, 1936, in the space of a few hours he developed aphasia and right hemiplegia and passed into coma. At operation a very large infiltrating glioma of the left temporal lobe was exposed, but could be only partially removed.

A single woman, aged 55, had a generalized convulsion which occurred without warning in May, 1935. Two further attacks occurred during the next six months. She also complained of occasional pains at the top of the head, but headache was not severe. Her memory became unreliable, and she occasionally had difficulty with her speech. On examination her ocular fundi were normal and the only abnormalities in her nervous system were slight weakness of the right lower face and of the grip of the right hand. $\mathrm{X}$-rays of the skull showed a slightly opaque, oval area, which appeared to be deep in 
the head in the middle line. Further X-rays after the introduction of air into the ventricle demonstrated a tumour of the corpus callosum displacing the lateral ventricles downwards and outwards. The tumour proved to be a glioma, which could be only partially removed at operation.

\section{Neuro-syphilis.}

Epilepsy occurs more frequently in general paralysis of the insane than in meningo-vascular syphilis. It may be the first symptom of either form of neurosyphilis. A careful clinical examination will usually reveal the characteristic abnormalities in the nervous system but, especially in meningo-vascular syphilis, epilepsy sometimes occurs in the absence of any neurological abnormality. It is important, therefore, that the blood Wassermann reaction should be examined in every case of epilepsy beginning in adult life, for which no cause is apparent, and if there is reason to suspect neurosyphilis the cerebro-spinal fluid should be examined also.

\section{Cardiovascular Disease.}

Cardiovascular disease may be responsible for epilepsy in several ways. Cerebral arteriosclerosis is the commonest cause of epileptic attacks after the age of 50. Minor attacks are seen rather more frequently than major, and either may occur in the absence of signs of focal vascular lesion of the brain, such as hemiplegia.

Stokes-Adams syndrome is not very common and for this reason may be missed as a cause of epilepsy. Since the usual cause of heart block is coronary atheroma Stokes-Adams syndrome is usually seen in the second half of life. The attacks caused by the defective circulation range in severity from brief loss of consciousness to a major epileptic convulsion. They may occur several times a day, especially during the phase of incomplete heart block, and do not necessarily disappear if heart block becomes complete. If the patient is first seen while the heart block is incomplete the heart rate and rhythm may be normal or there may be only occasional dropped beats. At this stage the diagnosis of the attack may be difficult and can be confirmed only by an electrocardiogram. When heart block is complete and the slow ventricular rate is established the diagnosis is easy. Other cardiac causes of enilepsy are rare, though I have seen epilepsy associated with mitral stenosis more often than can be explained on a chance basis.

The recognition that the carotid sinus plays an important part in the regulation of the circulation has thrown light upon a variety of attacks the nature of which was previously obscure. Pressure upon the internal carotid artery at its origin from the common carotid either by a tumour, enlarged gland, or even in susceptible individuals by the fingers or a tight collar, may, it is now known, cause attacks of giddiness, syncope or even epilepsy. The diagnosis of the nature of these attacks is based upon the ability to reproduce them by pressure in the region of the carotid sinus and their abolition after the application of a local anæsthetic to this area.

\section{Cerebral Cysticercosis.}

The cysticercus stage of tænia solium may occur in man and since the embryos have a predilection for the grey matter of the brain, epilepsy is a common symptom of this condition. Cysticercosis epilepsy is most often seen in soldiers who have lived in the tropics, especially in India, but the condition may occur in persons 
who have never been out of England. Patients with cerebral cysticercosis usually exhibit cysts in other parts of the body. Those in the subcutaneous tissues and in the muscles are firm oval bodies, usually rather smaller than a pea. When the parasites die the cysts become calcified and can then be demonstrated by radiography, but calcification usually occurs rather late in the brain. In a suspected case, therefore, the skin and muscles are carefully palpated for cysts, and if these are found one is excised for histological examination. Radiograms of the skull, shoulders and thighs should be taken and searched for calcified cysts. There are usually no abnormal physical signs in the nervous system.

\section{Traumatic Epilepsy.}

The rôle of trauma in the causation of epilepsy is not yet completely understood. Only about 5 per cent. of men suffering from gunshot wounds of the head during the Great War became epileptic. The site of the injury appears unimportant, but it has been shown that epilepsy is much more likely to occur if the wound penetrates the dura mater than if this is uninjured. There is evidence that inherited predisposition may play an important part in determining the occurrence of epilepsy after a head injury and it seems probable that those cases in which epilepsy follows a relatively slight injury may be explained in this way. The latent interval between the injusy and the onset of the attack is usually less than two years, but may be much longer. When epilepsy follows a severe head injury encephalograms should be taken since they may demonstrate the presence of a scar in the brain causing distortion of the ventricular system by traction. In such cases surgical treatment may be considered.

\section{Chronic Alcoholism.}

Chronic alcoholism is not a very common cause of epilepsy but may give rise to difficulties in diagnosis in the case of a patient who manages to conceal his tendency to alcoholic excess. The diagnosis is important because the prognosis of the epilepsy depends upon the successful treatment of the alcohol addiction.

\section{Idiopathic Epilepsy.}

When all other causes have, as far as possible, been eliminated there will remain patients whose attacks may begin at any age and who must be placed in the group of sufferers from idiopathic epilepsy. Nevertheless we do well to recognize that the use of the term idiopathic is a confession of our ignorance of the true cause of the attacks and we should only adopt it when we are satisfied that the most thorough investigation has left us no alternative. Even in such cases time, the great diagnostician, will sometimes prove us wrong.

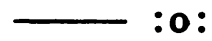

